\title{
Preliminary Results on the Experimental Investigation of the Structure Functions of Bound Nucleons
}

\author{
A. Bodek* \\ for Jefferson Lab experiments E02-109, E04-001, and E06-009. \\ Department of Physics and Astronomy, University of Rochester, Rochester, NY 14627, USA \\ E-mail: bodek@pas.rochester.edu
}

\begin{abstract}
We present preliminary results on an experimental study of the nuclear modification of the longitudinal $\left(\sigma_{L}\right)$ and transverse $\left(\sigma_{T}\right)$ structure functions of nucleons bound in nuclear targets. The origin of these modifications (commonly referred as as the EMC effect) is not fully understood. Our measurements of $\mathrm{R}=\sigma_{L} / \sigma_{T}$ for nuclei $\left(R_{A}\right)$ and for deuterium $\left(R_{D}\right)$ indicate that nuclear modifications of the structure functions of bound nucleons are different for the longitudinal and transverse structure functions, and that contrary to expectation from several theoretical models, $R_{A}<R_{D}$.
\end{abstract}

The XXIII International Workshop on Deep Inelastic Scattering and Related Subjects

April 27 - May 1, 2015

Southern Methodist University

Dallas, Texas 75275

\footnotetext{
* Speaker.
} 
In 1983, muon (EMC[1]) and electron (MIT-SLAC[2]) scattering experiments on iron discovered that the quark distributions in the nucleon are modified when the nucleon is bound in a nucleus. Subsequent experiments[3],[4] have investigated the effect for a a range of nuclei. The origin of these modifications is still not fully understood.[5, 6].

We investigate[7] these modifications though a high precision experimental study of both the longitudinal $\left(\sigma_{L}\right)$ and transverse $\left(\sigma_{T}\right)$ structure functions in electron scattering on deuterium (C), carbon $(\mathrm{C})$, aluminum $(\mathrm{Al})$, iron $(\mathrm{Fe})$, and copper $(\mathrm{Cu})$ nuclei. These data were taken by by several different experiments (See references [8],[9],[10],[11]) which cover a range in the square of the invariant momentum tansfer $\left(Q^{2}\right)$ and energy transfer $(v)$ to the nucleon, spanning hadronic final state invariant mass $W\left(W^{2}=M^{2}+2 M v-Q^{2}\right)$ from the resonance region $(\mathrm{W}<2 \mathrm{GeV})$ to the inelastic continuum $(\mathrm{W}>2 \mathrm{GeV})$.

The differential cross section for scattering of an unpolarized charged lepton with $E_{0}$, final energy $E^{\prime}$ and scattering angle $\theta$ can be written in terms of the structure functions $\mathscr{F}_{1}$ and $\mathscr{F}_{2}$ as:

$\frac{d^{2} \sigma}{d \Omega d E^{\prime}}\left(E_{0}, E^{\prime}, \theta\right)=\frac{4 \alpha^{2} E^{\prime 2}}{Q^{4}} \cos ^{2}(\theta / 2) \times\left[\mathscr{F}_{2}\left(x, Q^{2}\right) / v+2 \tan ^{2}(\theta / 2) \mathscr{F}_{1}\left(x, Q^{2}\right) / M\right]$

where $\alpha$ is the fine structure constant, $M$ is the nucleon mass, $v=E_{0}-E^{\prime}$, and $Q^{2}=4 E_{0} E^{\prime} \sin ^{2}(\theta / 2)$. Within the quark parton model, the target mass scaling variable[14] $\xi_{T M}=\frac{Q^{2}}{M v\left[1+\sqrt{1+Q^{2} / v^{2}}\right]}$ is the fractional momentum (parallel to the direction of the momentum transfer) carried by the struck parton in the nucleon. At large $Q^{2}, \xi_{T M}=x$ where $x=Q^{2} / 2 M v$ is the Bjorken scaling variable.

In Quantum Chromodynamics (QCD) $\mathscr{F}_{2}\left(x, Q^{2}\right)$ is expressed in terms of charge weighted sums of the fractional momentum distributions of quarks and antiquarks in the nucleon. The ratio of $\mathscr{F}_{2}$ for a nuclear target $\left(\mathscr{F}_{2 A}\right)$ to $\mathscr{F}_{2}$ for deuterium $\left(\mathscr{F}_{2 D}\right)$ is a function of $x$. The $x<0.1$ region, where $\mathscr{F}_{2 A} / \mathscr{F}_{2 D}<1$, is known as the shadowing region. The $0.1<x<0.3$ region, where $\mathscr{F}_{2 A} / \mathscr{F}_{2 D}>1$, is known as the anti-shadowing region. The $0.3<x<0.9$ region, where $\mathscr{F}_{2 A} / \mathscr{F}_{2 D}<1$, is referred to as the region of the "EMC Effect", and the $x>0.9$ region, where $\mathscr{F}_{2 A} / \mathscr{F}_{2 D}>1$, is dominated by Fermi motion. In our studies, we focus on the region of the "EMC Effect", $0.3<x<0.9$, where the data indicate that there is softening of the fractional momentum distribution of quarks for nucleons bound in the nucleus

Alternatively, one can view this scattering process in terms of the cross section for the absorption of transverse $\left(\sigma_{T}\right)$ and longitudinal $\left(\sigma_{L}\right)$ virtual photons, where

$$
\frac{d^{2} \sigma}{d \Omega d E^{\prime}}=\Gamma\left[\sigma_{T}\left(x, Q^{2}\right)+\varepsilon \sigma_{L}\left(x, Q^{2}\right)\right] .
$$

Here $K=\frac{2 M v-Q^{2}}{2 M}$, and $\Gamma=\frac{\alpha K E^{\prime}}{4 \pi^{2} Q^{2} E_{0}}\left(\frac{2}{1-\varepsilon}\right)$ is the flux and $\varepsilon=\left[1+2\left(1+\frac{Q^{2}}{4 M^{2} x^{2}}\right) \tan ^{2} \frac{\theta}{2}\right]^{-1}$ is the degree of longitudinal polarization of the virtual photons. The structure functions $\mathscr{F}_{1}$ and $\mathscr{F}_{2}$ are proportional to $\sigma_{T}$, and $\left[\sigma_{T}+\sigma_{L}\right]$, respectively. Here, $\mathscr{R}=\sigma_{L} / \sigma_{T}$ is given by:

$$
\mathscr{R}\left(x, Q^{2}\right)=\frac{\sigma_{L}}{\sigma_{T}}=\frac{\mathscr{F}_{2}}{2 x \mathscr{F}_{1}}\left(1+\frac{4 M^{2} x^{2}}{Q^{2}}\right)-1=\frac{\mathscr{F}_{L}}{2 x \mathscr{F}_{1}}
$$

Contributions to $R$ originate from the perpendicular component of the momentum of the quarks[12] with respect to momentum transfer vector. A perpendicular momentum component can originated from QCD gluon emission[13] $\left(R_{Q C D}\right)$, from quark binding in the nucleon (target mass 
corrections[14] $\left(R_{T M}\right)$, and from non-perturbative processes such as interactions with more than one quark (higher twist). Numerically, $R_{Q C D}$ dominates at small $x, R_{T M}$ dominates at large $x$, and higher twist effects are important at very small $Q^{2}$ (since $R$ must be zero at $Q^{2}=0$ ). Nuclear binding can modify both the parallel and perpendicular momentum distributions of quarks, antiquarks and gluons of bound nucleons thus modifying both $F_{2}$ and $R$.

Here, we report on some preliminary results[7] on the data[15] taken in 2007. These data were taken with $\mathrm{D}, \mathrm{C}, \mathrm{Al}, \mathrm{Fe}$ and $\mathrm{Cu}$ targets for $Q^{2} 2,3$ and $3.7 \mathrm{GeV}^{2}$. Since quark-hadron duality has been shown to be valid[16] in this region, we can investigate the EMC effect at large $\xi_{T M}$ by extending the studies to the resonance region. Incident electrons at seven different energies provided by the Jefferson Lab accelerator are scattered from a 4-cm-long liquid deuterium target, and solid nuclear targets. Electrons are detected in the Hall C High Momentum Spectrometer (HMS) at angle settings ranging from $12^{0}$ to $75^{\circ}$. The charge symmetric (CS) backgrounds from the $e^{+} e^{-}$conversion of photons from $\pi^{0}$ production and its subsequent positrons are measured in runs with reverse polarity. Background from eletro-produced charged pions are identified and removed by using both a gas Cherenkov counter and an electromagnetic calorimeter. Events scattering from the walls (Al) of the cryogenic target cell are subtracted by measuring the scattering from an empty target replica. For additional details regarding the analysis and Hall $\mathrm{C}$ apparatus employed in this experiment, see Refs. [7] and [17].

The differential cross sections are determined from the electron rates after correcting for inefficiencies, background and radiative corrections (which include bremsstrahlung, vertex corrections and loop diagrams standard to electron scattering experiments). The cross sections are interpolated to fixed $Q^{2}$ values of 2, 3 and $4 \mathrm{GeV}^{3}$, in bins of $W$. The longitudinal and transverse cross sections and structure functions are extracted from linear fits (equation 1) to cross section measurements spanning a range of $\varepsilon(0.2<\Delta \varepsilon<0.6)$ for fixed values of $W$ and $Q^{2}$.

Overall normalization errors cancel in the extraction of $R_{D}$. The overall $1.4 \%$ systematic error[7] in the $\varepsilon$ dependence of the cross that originates from $E_{0}(0.25 \%)$, beam charge $(0.3 \%)$, efficiencies $(0.35 \%)$, CS background (0.1-0.4\%), acceptance $(0.7 \%)$ and radiative corrections $(<1 \%)$, yields a systematic uncertainty in $R_{D}$ of \pm 0.028 . At present, there is no reliable calculation of twophoton exchange contributions to the radiative corrections. The two photon exchange contribution to the cross section for a point like proton has been estimated[18] to be $+0.3 \%$ at $\varepsilon=1$ and $+2.3 \%$ at $\varepsilon=0$. If we assume that the contributions for inelastic scattering from point like quarks is similar to a point like proton, the two-photon contribution changes $F_{2 D}$ by $+0.3 \%$ and $R_{D}$ by +0.04 .

For targets with atomic number $Z>1$ these coulomb corrections [7] account for the effect of the electric field of the additional protons on the incident and scattered electrons. The additional protons create an electrostatic potential $V(r)=-\frac{3 \alpha(Z-1)}{2 R}+\frac{r \alpha(Z-1)}{2 R^{2}}\left(R=1.1 A^{1 / 3}+0.775 A^{-1 / 3}\right.$ for atomic weight $\mathrm{A}$ ). It has been shown[19] that in the effective momentum approximation (EMA) the effective potential $V_{e f f}=0.8 V(r=0)$ yields results which are in good agreement with the full DWBA calculation[20]. This value for $V_{\text {eff }}$ also agrees with values extracted from a comparison of positron and electron quasi elastic scattering cross sections[21]. The effective incident energy is $E_{e f f}=E_{0}+V_{e f f}$, and the effective scattered energy is $E_{e f f}^{\prime}=E^{\prime}+V_{e f f}$. In addition, there is a focusing factor $F_{f o c}=\frac{E_{0}+V_{e f f}}{E_{0}}$. A model of the differential cross section $\left(\sigma_{\text {model }}\right)$ is used to correct 
the measured cross sections $\sigma_{\text {meas }}$ and yield a coulomb corrected(CC) $\sigma_{\text {meas }}^{c c}$ :

$$
\sigma_{\text {meas }}^{c c}\left(E_{0}, E^{\prime}\right)=\frac{\sigma_{\text {meas }}\left(E_{0}, E^{\prime}\right) \sigma_{\text {model }}\left(E_{0}, E^{\prime}\right)}{\sigma_{\text {model }}\left(E_{\text {eff }}, E_{\text {eff }}^{\prime}\right) F_{\text {foc }}^{2}}
$$

where the effective $Q^{2}$ is $Q_{e f f}^{2}=4\left(E_{0}+V_{e f f}\right)\left(E^{\prime}+V_{e f f}\right) \sin ^{2}(\theta / 2)$.

\section{RC-RD}
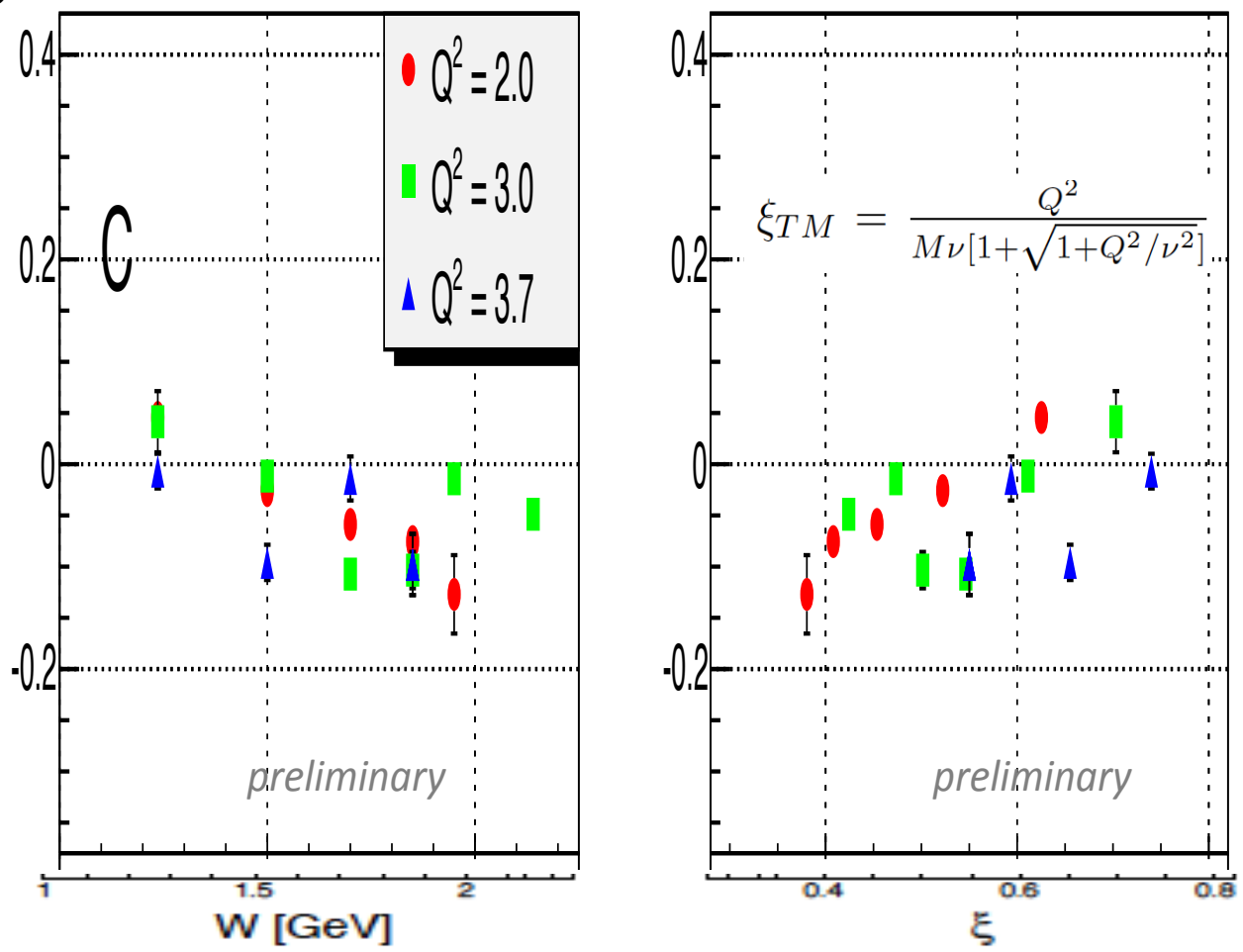

Figure 1: Extracted values of $R_{A}-R_{D}$ for carbon versus W (left panel) and versus $\xi_{T M}$ (right panel). The three values of $Q^{2}$ are shown as different symbols. Statistical errors only. On average $R_{C}<R_{D}$, which is contrary to expectation from several theoretical models.

The difference $R_{D}-R_{A}$ and $F_{2 D} / F_{2 A}$ are extracted from linear fits to the ratio of differential cross section.

$$
\frac{\sigma_{D}}{\sigma_{A}}=\frac{\sigma_{D}^{T}}{\sigma_{A}^{T}}\left[1+\varepsilon^{\prime}\left(R_{D}-R_{A}\right)\right]
$$

where $\varepsilon^{\prime}=\varepsilon /\left(1+\varepsilon R_{A}\right)$. Note that $\varepsilon R_{A}$ is small and the resonance structure in $R_{A}$ is smeared by Fermi motion. Therefore, the extracted $R_{D}-R_{A}$ values are insensitive to the most of the systematic errors listed above for $R_{D}$. For this study, we bin the data in wide bins of $W$.

Fig. 1 shows the results expressed in terms of $R_{C}-R_{D}$ for Carbon versus $W$ (left panel) and versus $\xi_{T M}$ (right panel). The three values of $Q^{2}$ are shown in different symbols. For $W>1.4 \mathrm{GeV}$ the average value for $Q^{2}=2,3$, and $4 \mathrm{GeV}^{2}$ is: $\left\langle R_{C}-R_{D}\right\rangle=-0.047 \pm 0.006$ (statistical error only).

One possible source of systematic error in $R_{A}-R_{D}$ is two-photon exchange where the two photons scatter from quarks in different nucleons in the nucleus. Some, but not all of the contribution from interactions involving one hard photon absorbed by one nucleon and multiple soft photons 
absorbed by other nucleons in the nucleus are included in the Coulomb correction. In addition, the exchange of one hard photon with a quark in one nucleon, and another hard photon with a quark in another nucleon is not included. Theoretical estimates of the contribution of this process are not presently available.

Our result that $R_{A}<R_{D}$ is contrary to several theoretical expectations. Calculations [22] of the effect of the Fermi motion on nucleons in nuclei predict a small difference in the opposite direction to what we observe. A decrease of the gluon distributions in nuclei yields $R_{A}<R_{D}$ in the $x<0.1$ shadowing region. However, the contribution of the gluon distributions to $R$ for $x>0.3$ is small and these models[23] predict $R_{A}=R_{D}$ for the $x$ region of our measurements (i.e. the EMC effect region). Models[24] which attribute the EMC effect to the presence of pions in nuclei also predict an effect in the opposite direction to what we observe (these models also predict enhancement of antiquarks in the nucleus which has been ruled out by Drell-Yan experiments on nuclear targets[5]).

In the naive quark parton model[12], $R=4\left\langle K_{T}^{2}\right\rangle / Q^{2}$, where $K_{T}$ is the momentum of quarks in a nucleon perpendicular to the direction of the momentum transfer vector. Therefore, a possible interpretation of the data is that $\left\langle K_{T}^{2}\right\rangle$ is smaller for bound nucleons, and that the distributions for both the parallel and perpendicular momentum components of quarks in a nucleon are softened in a nuclear medium due to partial deconfinement[25] of quarks caused by short range nucleon-nucleon correlations (SRC) in nuclei[26].

In summary, our preliminary measurements of $\mathrm{R}=\sigma_{L} / \sigma_{T}$ for Carbon $\left(R_{C}\right)$ and for deuterium $\left(R_{D}\right)$ indicate that nuclear modifications of the structure functions of bound nucleons are different for the longitudinal and transverse structure functions, and that contrary to expectation from several theoretical models, $R_{C}<R_{D}$.

\section{References}

[1] J. J. Aubert et al (EMC-NA2 collaboration), Phys. Lett. 12B, 275 (1983).

[2] A. Bodek et al. (SLAC-E87), Phys. Rev. Lett. 50.1431 (1983); Phys. Rev, Lett 51, 534 (1983).

[3] S. Dasu, et al., (SLAC-E140), Phys. Rev. Lett. 61, 1061 (1988); Phys. Rev. D49, 5641 (1994).

[4] G. Gomez et al. (SLAC-E139), Phys. Rev. D49, 4348 (1994).

[5] G. A. Miller, Eur.Phys. J. A 31, 578 (2007).

[6] J. Arrington, J. Phys. Conf. Ser. 69, 012024 (2007).

[7] V. Mamyan, Ph.D. Thesis, U. Virginia, 2010. arXiv:1202.1457.

[8] https://hallcweb.jlab.org/experiments/rosen07/

[9] Jlab E02-109 and E06-009 (D) M. E. Christy, C. Keppel, spokespersons.

[10] Jlab E04-001(C, Al, Fe, Cu) A. Bodek, C. Keppel, spokespersons.

[11] Jlab E06-009 (D) M. E. Christy, C. Keppel, spokespersons.

[12] R. P. Feynman, Photon-Hadron Interactions (Benjamin, Reading, MA,1972), pp. 138-139

[13] A.. D. Martin, R. G. Roberts, W. J. Stirling, R. S. Thorne. Eur. Phys. J. C18, 117 (2000).

[14] R. Barbieri, J. R. Ellis, M. K. Gaillard and G. G. Ross, Nucle Phys. B117, 50 (1976); H. Georgi and H. D. Politzer, Phys. Rev. D14, 1829 (1976). 
[15] In 2007, high $Q^{2}$ data were taken by experiments E02-109, E04-001 and E06-009 at the following energies and scattering angles: $2.1 \mathrm{GeV},\left(45^{0}, 60^{0}, 75^{0}\right) 3.12 \mathrm{GeV},\left(15^{0}, 25^{0}, 35^{0}, 45^{0}, 60^{0}, 75^{0}\right) 4.07$ $\mathrm{GeV},\left(43^{0}, 50^{0}, 60^{0}, 76^{0}\right), 3.27 \mathrm{GeV},\left(22^{0}, 33^{0}, 43^{0}, 58^{0}, 75^{0}\right), 4.13 \mathrm{GeV},\left(39^{0}, 48^{0}, 58^{0}, 75^{0}\right), 4.2$ $\mathrm{GeV}$,(Al only) $\left(13^{0}, 21^{0}, 33.7^{0}\right)$, and $5.15 \mathrm{GeV}\left(12^{0}, 18^{0}, 23^{0}, 27^{0}, 30^{0}, 33^{0}, 38^{0}\right)$.

[16] W. Melnitchouk, R. Ent, and C. E. Keppel, Phys, Rep. 406, 127 (2005).

[17] M. E. Christy et al. [E94110 collaboration] Phys. Rev. C70, 015206 (2004).

[18] J. Arrington, P.G. Blunden, and W. Melnitchouk. Prog.Part.Nucl.Phys. 66, 782 (2011) arXiv:1105.0951.

[19] A. Aste, C. von Arx and D. Trautmann, Eur. Phys. J. A 26, 167 (2005).

[20] Y. Jin, D. S. Onley, and L. E. Wright, Phys. Rev. C50, 168 (1994).

[21] P. Gueye et al., Phys. Rev. C 60, 044308 (1999).

[22] M. Erickson, S. Kumano, Phys. Rev. C, 022201 (2003).

[23] N. Armesto et. al. Phys. Lett. B694, 38 (2010).

[24] G. A. Miller, Phys. Rev. C 64, 022201 (2001).

[25] Partial deconfinement of quarks in low momentum nucleons in nuclei has been ruled out by measurements of the form factors of bound nucleons, but not for high momentum nucleons with high virtuality from SRC.

[26] L. B. Weinstein et al. Phys. Rev. Lett. 106, 052301 (2011) 